\title{
ERGODIC THEORY: RECURRENCE
}

\author{
NIKOS FRANTZIKINAKIS AND RANDALL MCCUTCHEON
}

\section{CONTEnts}

1. Definition of the Subject and its Importance 3

2. Introduction 3

3. Quantitative Poincaré Recurrence 5

4. Subsequence Recurrence 7

5. Multiple Recurrence 11

6. Connections with Combinatorics and Number Theory 14

7. Future Directions 17

References $\quad 19$

\begin{abstract}
Almost every, essentially: Given a Lebesgue measure space $(X, \mathcal{B}, \mu)$, a property $P(x)$ predicated of elements of $X$ is said to hold for almost every $x \in X$, if the set $X \backslash\{x: P(x)$ holds $\}$ has zero measure. Two sets $A, B \in \mathcal{B}$ are essentially disjoint if $\mu(A \cap B)=0$.

Conservative system: Is an infinite measure preserving system such that for no set $A \in \mathcal{B}$ with positive measure are $A, T^{-1} A, T^{-2} A, \ldots$ pairwise essentially disjoint.

$\left(c_{n}\right)$-conservative system: If $\left(c_{n}\right)_{n \in \mathbb{N}}$ is a decreasing sequence of positive real numbers, a conservative ergodic measure preserving transformation $T$ is $\left(c_{n}\right)$-conservative if for some non-negative function $f \in L^{1}(\mu)$, $\sum_{n=1}^{\infty} c_{n} f\left(T^{n} x\right)=\infty$ a.e.

Doubling map: If $\mathbb{T}$ is the interval $[0,1]$ with its endpoints identified and addition performed modulo 1 , the (non-invertible) transformation $T: \mathbb{T} \rightarrow \mathbb{T}$, defined by $T x=2 x \bmod 1$, preserves Lebesgue measure, hence induces a measure preserving system on $\mathbb{T}$.

Ergodic system: Is a measure preserving system $(X, \mathcal{B}, \mu, T)$ (finite or infinite) such that every $A \in \mathcal{B}$ that is $T$-invariant (i.e. $T^{-1} A=A$ ) satisfies either $\mu(A)=0$ or $\mu(X \backslash A)=0$. (One can check that the rotation $R_{\alpha}$ is ergodic if and only if $\alpha$ is irrational, and that the doubling map is ergodic.) Ergodic decomposition: Every measure preserving system $(X, \mathcal{X}, \mu, T)$ can be expressed as an integral of ergodic systems; for example, one can
\end{abstract}

2000 Mathematics Subject Classification. Primary: 37A99; Secondary: 37A45, 28D05. The first author was partially supported by NSF grant DMS-0701027. 
write $\mu=\int \mu_{t} d \lambda(t)$, where $\lambda$ is a probability measure on $[0,1]$ and $\mu_{t}$ are $T$ invariant probability measures on $(X, \mathcal{X})$ such that the systems $\left(X, \mathcal{X}, \mu_{t}, T\right)$ are ergodic for $t \in[0,1]$.

Ergodic theorem: States that if $(X, \mathcal{B}, \mu, T)$ is a measure preserving system and $f \in L^{2}(\mu)$, then $\lim _{N \rightarrow \infty}\left\|\frac{1}{N} \sum_{n=1}^{N} T^{n} f-P_{f}\right\|_{L^{2}(\mu)}=0$, where $P_{f}$ denotes the orthogonal projection of the function $f$ onto the subspace $\left\{f \in L^{2}(\mu): T f=f\right\}$.

Hausdorff $a$-measure: Let $(X, \mathcal{B}, \mu, T)$ be a measure preserving system endowed with a $\mu$-compatible metric $d$. The Hausdorff $a$-measure $\mathcal{H}_{a}(X)$ of $X$ is an outer measure defined for all subsets of $X$ as follows: First, for $A \subset X$ and $\varepsilon>0$ let $\mathcal{H}_{a, \varepsilon}(A)=\inf \left\{\sum_{i=1}^{\infty} r_{i}^{a}\right\}$, where the infimum is taken over all countable coverings of $A$ by sets $U_{i} \subset X$ with diameter $r_{i}<\varepsilon$. Then define $\mathcal{H}_{a}(A)=\lim \sup _{\varepsilon \rightarrow 0} \mathcal{H}_{a, \varepsilon}(A)$.

Infinite measure preserving system: Same as measure preserving system, but $\mu(X)=\infty$.

Invertible system: Is a measure preserving system $(X, \mathcal{B}, \mu, T)$ (finite or infinite), with the property that there exists $X_{0} \in X$, with $\mu\left(X \backslash X_{0}\right)=0$, and such that the transformation $T: X_{0} \rightarrow X_{0}$ is bijective, with $T^{-1}$ measurable. Measure preserving system: Is a quadruple $(X, \mathcal{B}, \mu, T)$, where $X$ is a set, $\mathcal{B}$ is a $\sigma$-algebra of subsets of $X$ (i.e. $\mathcal{B}$ is closed under countable unions and complementation), $\mu$ is a probability measure (i.e. a countably additive function from $\mathcal{B}$ to $[0,1]$ with $\mu(X)=1$ ), and $T: X \rightarrow X$ is measurable (i.e. $T^{-1} A=\{x \in X: T x \in A\} \in \mathcal{B}$ for $A \in \mathcal{B}$ ), and $\mu$-preserving (i.e. $\left.\mu\left(T^{-1} A\right)=\mu(A)\right)$. Moreover, throughout the discussion we assume that the measure space $(X, \mathcal{B}, \mu)$ is Lebesgue (see Section 1.0 of [3]).

$\mu$-compatible metric: Is a separable metric on $X$, where $(X, \mathcal{B}, \mu)$ is a probability space, having the property that open sets measurable.

Positive definite sequence: Is a complex-valued sequence $\left(a_{n}\right)_{n \in \mathbb{Z}}$ such that for any $n_{1}, \ldots, n_{k} \in \mathbb{Z}$ and $z_{1}, \ldots, z_{k} \in \mathbb{C}, \sum_{i, j=1}^{k} a_{n_{i}-n_{j}} z_{i} \overline{z_{j}} \geq 0$.

Rotations on $\mathbb{T}$ : If $\mathbb{T}$ is the interval $[0,1]$ with its endpoints identified and addition performed modulo 1 , then for every $\alpha \in \mathbb{R}$ the transformation $R_{\alpha}: \mathbb{T} \rightarrow \mathbb{T}$, defined by $R_{\alpha} x=x+\alpha$, preserves Lebesgue measure on $\mathbb{T}$ and hence induces a measure preserving system on $\mathbb{T}$.

Syndetic set: Is a subset $E \subset \mathbb{Z}$ having bounded gaps. If $G$ is a general discrete group, a set $E \subset G$ is syndetic if $G=F E$ for some finite set $F \subset G$. Upper density: Is the number $\bar{d}(\Lambda)=\limsup _{N \rightarrow \infty} \frac{|\Lambda \cap\{-N, \ldots, N\}|}{2 N+1}$, where $\Lambda \subset \mathbb{Z}$ (assuming the limit to exist). Alternatively for measurable $E \subset \mathbb{R}^{m}$, $\bar{D}(E)=\lim \sup _{l(S) \rightarrow \infty} \frac{m(S \cap E)}{m(S)}$, where $S$ ranges over all cubes in $\mathbb{R}^{m}$, and $l(S)$ denotes the length of the shortest edge of $S$.

Notation: The following notation will be used throughout the article: $T f=$ $f \circ T,\{x\}=x-[x], \mathrm{D}-\lim _{n \rightarrow \infty}\left(a_{n}\right)=a \leftrightarrow \bar{d}\left(\left\{n:\left|a_{n}-a\right|>\varepsilon\right\}\right)=0$ for every $\varepsilon>0$. 


\section{Definition of the Subject and its Importance}

The basic principle that lies behind several recurrence phenomena, is that the typical trajectory of a system with finite volume comes back infinitely often to any neighborhood of its initial point. This principle was first exploited by Poincaré in his 1890 King Oscar prize-winning memoir that studied planetary motion. Using the prototype of an ergodic theoretic argument, he showed that in any system of point masses having fixed total energy that restricts its dynamics to bounded subsets of its phase space, the typical state of motion (characterized by configurations and velocities) must recur to an arbitrary degree of approximation.

Among the recurrence principle's more spectacularly counterintuitive ramifications is that isolated ideal gas systems that do not lose energy will return arbitrarily closely to their initial states, even when such a return entails a decrease in entropy from equilibrium, in apparent contradiction to the second law of thermodynamics. Such concerns, previously canvassed by Poincaré himself, were more infamously expounded by Zermelo ([116]) in 1896. Subsequent clarifications by Boltzmann, Maxwell and others led to an improved understanding of the second law's primarily statistical nature. (For an interesting historical/philosophical discussion, see [106]; also [11. For a probabilistic analysis of the likelihood of observing second law violations in small systems over short time intervals, see [42.)

These discoveries had a profound impact in dynamics, and the theory of measure preserving transformations (ergodic theory) evolved from these developments. Since then, the Poincaré recurrence principle has been applied to a variety of different fields in mathematics, physics, and information theory. In this article we survey the impact it has had in ergodic theory, especially as pertains to the field of ergodic Ramsey theory. (The heavy emphasis herein on the latter reflects authorial interest, and is not intended to transmit a proportionate image of the broader landscape of research relating to recurrence in ergodic theory.) Background information we assume in this article can be found in the books [41, 60, 68, 81, 100, 112] (see also the article "Measure Preserving Systems" by K. Petersen in this volume). Related information can also be found on the survey articles [10, 12, 13, 47, 84, 85, 86].

\section{INTRODUCTION}

In this section we shall give several formulations of the Poincare recurrence principle using the language of ergodic theory. Roughly speaking, the principle states that in a finite (or conservative) measure preserving system, every set of positive measure (or almost every point) comes back to itself infinitely many times under iteration. Despite the profound importance of these results, their proofs are extremely simple.

Theorem 2.1 (Poincaré Recurrence for Sets). Let $(X, \mathcal{B}, \mu, T)$ be a measure preserving system and $A \in \mathcal{B}$ with $\mu(A)>0$. Then $\mu\left(A \cap T^{-n} A\right)>0$ for infinitely many $n \in \mathbb{N}$. 
Proof. Since $T$ is measure preserving, the sets $A, T^{-1} A, T^{-2} A, \ldots$ have the same measure. These sets cannot be pairwise essentially disjoint, since then the union of finitely many of them would have measure greater than $\mu(X)=$ 1. Therefore, there exist $m, n \in \mathbb{N}$, with $n>m$, such that $\mu\left(T^{-m} A \cap\right.$ $\left.T^{-n} A\right)>0$. Again since $T$ is measure preserving, we conclude that $\mu(A \cap$ $\left.T^{-k} A\right)>0$, where $k=n-m>0$. Repeating this argument for the iterates $A, T^{-m} A, T^{-2 m} A, \ldots$, for all $m \in \mathbb{N}$, we easily deduce that $\mu\left(A \cap T^{-n} A\right)>0$ for infinitely many $n \in \mathbb{N}$.

We remark that the above argument actually shows that $\mu\left(A \cap T^{-n} A\right)>0$ for some $n \leq\left[\frac{1}{\mu(A)}\right]+1$.

Theorem 2.2 (Poincaré Recurrence for Points). Let $(X, \mathcal{B}, \mu, T)$ be a measure preserving system and $A \in \mathcal{B}$. Then for almost every $x \in A$ we have that $T^{n} x \in A$ for infinitely many $n \in \mathbb{N}$.

Proof. Let $B$ be the set of $x \in A$ such that $T^{n} x \notin A$ for all $n \in \mathbb{N}$. Notice that $B=A \backslash \bigcup_{n \in \mathbb{N}} T^{-n} A$; in particular, $B$ is measurable. Since the iterates $B, T^{-1} B, T^{-2} B, \ldots$ are pairwise essentially disjoint, we conclude (as in the proof of Theorem 2.1) that $\mu(B)=0$. This shows that for almost every $x \in A$ we have that $T^{n} x \in A$ for some $n \in \mathbb{N}$. Repeating this argument for the transformation $T^{m}$ in place of $T$ for all $m \in \mathbb{N}$, we easily deduce the advertised statement.

Next we give a variation of Poincaré recurrence for measure preserving systems endowed with a compatible metric:

Theorem 2.3 (Poincaré Recurrence for Metric Systems). Let $(X, \mathcal{B}, \mu, T)$ be a measure preserving system, and suppose that $X$ is endowed with a $\mu$ compatible metric. Then for almost every $x \in X$ we have

$$
\liminf _{n \rightarrow \infty} d\left(x, T^{n} x\right)=0 .
$$

The proof of this result is similar to the proof of Theorem 2.2 (see [60], page 61). Applying this result to the doubling map $T x=2 x$ on $\mathbb{T}$, we get that for almost every $x \in X$, every string of zeros and ones in the dyadic expansion of $x$ occurs infinitely often.

We remark that all three formulations of the Poincaré Recurrence Theorem that we have given hold for conservative systems as well. See, e.g., [3] for details.

This article is structured as follows. In Section 3 we give a few quantitative versions of the previously mentioned qualitative results. In Sections 4 and 5 we give several refinements of the Poincaré recurrence theorem, by restricting the scope of the return time $n$, and by considering multiple intersections (for simplicity we focus on $\mathbb{Z}$-actions). In Section 6 we give various implications of the recurrence results in combinatorics and number theory (see also the article "Ergodic Theory: Interactions with Combinatorics and Number Theory" by T. Ward in the present volume). Lastly, in Section 7 we give several open problems related to the material presented in Sections 4.6. 


\section{Quantitative Poincaré Recurrence}

3.1. Early results. For applications it is desirable to have quantitative versions of the results mentioned in the previous section. For example one would like to know how large $\mu\left(A \cap T^{-n} A\right)$ can be made and for how many $n$.

Theorem 3.1 (Khintchine 90]). Let $(X, \mathcal{B}, \mu, T)$ be a measure preserving system and $A \in \mathcal{B}$. Then for every $\varepsilon>0$ we have $\mu\left(A \cap T^{-n} A\right)>\mu(A)^{2}-\varepsilon$ for a set of $n \in \mathbb{N}$ that has bounded gaps.

By considering the doubling map $T x=2 x$ on $\mathbb{T}$ and letting $A=\mathbf{1}_{[0,1 / 2)}$, it is easy to check that the lower bound of the previous result cannot be improved. We also remark that it is not possible to estimate the size of the gap by a function of $\mu(A)$ alone. One can see this by considering the rotations $R_{k} x=x+1 / k$ for $k \in \mathbb{N}$, defined on $\mathbb{T}$, and letting $A=\mathbf{1}_{[0,1 / 3]}$.

Concerning the second version of the Poincaré recurrence theorem, it is natural to ask whether for almost every $x \in X$ the set of return times $S_{x}=\left\{n \in \mathbb{N}: T^{n} x \in A\right\}$ has bounded gaps. This is not the case, as one can see by considering the doubling map $T x=2 x$ on $\mathbb{T}$ with the Lebesgue measure, and letting $A=\mathbf{1}_{[0,1 / 2)}$. Since Lebesgue almost every $x \in \mathbb{T}$ contains arbitrarily large blocks of ones in its dyadic expansion, the set $S_{x}$ has unbounded gaps. Nevertheless, as an easy consequence of the Birkhoff ergodic theorem ([27]), one has the following:

Theorem 3.2. Let $(X, \mathcal{B}, \mu, T)$ be a measure preserving system and $A \in \mathcal{B}$ with $\mu(A)>0$. Then for almost every $x \in X$ the set $S_{x}=\left\{n \in \mathbb{N}: T^{n} x \in A\right\}$ has well defined density and $\int d\left(S_{x}\right) d \mu(x)=\mu(A)$. Furthermore, for ergodic measure preserving systems we have $d\left(S_{x}\right)=\mu(A)$ a.e.

Another question that arises naturally is, given a set $A$ with positive measure and an $x \in A$, how long should one wait till some iterate $T^{n} x$ of $x$ hits $A$ ? By considering an irrational rotation $R_{\alpha}$ on $\mathbb{T}$, where $\alpha$ is very near to, but not less than, $\frac{1}{100}$, and letting $A=1_{[0,1 / 2]}$, one can see that the first return time is a member of the set $\{1,50,51\}$. So it may come as a surprise that the average first return time does not depend on the system (as long as it is ergodic), but only on the measure of the set $A$.

Theorem 3.3 (Kac [82]). Let $(X, \mathcal{B}, \mu, T)$ be an ergodic measure preserving system and $A \in \mathcal{B}$ with $\mu(A)>0$. For $x \in X$ define $R_{A}(x)=\min \{n \in$ $\left.\mathbb{N}: T^{n} x \in A\right\}$. Then for $x \in A$ the expected value of $R_{A}(x)$ is $1 / \mu(A)$, i.e. $\int_{A} R_{A}(x) d \mu=1$.

3.2. More Recent Results. As we mentioned in the previous section, if the space $X$ is endowed with a $\mu$-compatible metric $d$, then for almost every $x \in X$ we have that $\liminf _{n \rightarrow \infty} d\left(x, T^{n} x\right)=0$. A natural question is, how much iteration is needed to come back within a small distance of a given typical point? Under some additional hypothesis on the metric $d$ we have the following answer: 
Theorem 3.4 (Boshernitzan [28]). Let $(X, \mathcal{B}, \mu, T)$ be a measure preserving system endowed with a $\mu$-compatible metric $d$. Assume that the Hausdorff a-measure $\mathcal{H}_{a}(X)$ of $X$ is $\sigma$-finite (i.e., $X$ is a countable union of sets $X_{i}$ with $\left.\mathcal{H}_{a}\left(X_{i}\right)<\infty\right)$. Then for almost every $x \in X$,

$$
\liminf _{n \rightarrow \infty}\left\{n^{\frac{1}{a}} \cdot d\left(x, T^{n} x\right)\right\}<\infty .
$$

Furthermore, if $\mathcal{H}_{a}(X)=0$, then for almost every $x \in X$,

$$
\liminf _{n \rightarrow \infty}\left\{n^{\frac{1}{a}} \cdot d\left(x, T^{n} x\right)\right\}=0 .
$$

One can see from rotations by "badly approximable" vectors $\alpha \in \mathbb{T}^{k}$ that the exponent $1 / k$ in the previous theorem cannot be improved. Several applications of Theorem 3.4 to billiard flows, dyadic transformations, symbolic flows and interval exchange transformations are given in 28. For a related result dealing with mean values of the limits in Theorem 3.4 see [105].

An interesting connection between rates of recurrence and entropy of an ergodic measure preserving system was established by Ornstein and Weiss ([99]), following earlier work of Wyner and Ziv ([115]):

Theorem 3.5 (Ornstein \& Weiss [99]). Let $(X, \mathcal{B}, \mu, T)$ be an ergodic measure preserving system and $\mathcal{P}$ be a finite partition of $X$. Let $P_{n}(x)$ be the element of the partition $\bigvee_{i=0}^{n-1} T^{-i} \mathcal{P}=\left\{\bigcap_{i=0}^{n-1} T^{-i} P^{(i)}: P^{(i)} \in \mathcal{P}, 0 \leq i<n\right\}$ that contains $x$. Then for almost every $x \in X$, the first return time $R_{n}(x)$ of $x$ to $P_{n}(x)$ is asymptotically equivalent to $e^{h(T, \mathcal{P}) n}$, where $h(T, \mathcal{P})$ denotes the entropy of the system with respect to the partition $\mathcal{P}$. More precisely,

$$
\lim _{n \rightarrow \infty} \frac{\log R_{n}(x)}{n}=h(T, \mathcal{P}) .
$$

An extension of the above result to some classes of infinite measure preserving systems was given in [67.

Another connection of recurrence rates, this time with the local dimension of an invariant measure, is given by the next result:

Theorem 3.6 (Barreira [5]). Let $(X, \mathcal{B}, \mu, T)$ be an ergodic measure preserving system. Define the upper and lower recurrence rates

$$
\underline{R}(x)=\liminf _{r \rightarrow 0} \frac{\log \tau_{r}(x)}{-\log r} \quad \text { and } \quad \bar{R}(x)=\limsup _{r \rightarrow 0} \frac{\log \tau_{r}(x)}{-\log r},
$$

where $\tau_{r}(x)$ is the first return time of $T^{k} x$ in $B(x, r)$, and the upper and lower pointwise dimensions

$$
\underline{d}_{\mu}(x)=\liminf _{r \rightarrow 0} \frac{\log \mu(B(x, r))}{\log r} \quad \text { and } \quad \bar{d}_{\mu}(x)=\limsup _{r \rightarrow 0} \frac{\log \mu(B(x, r))}{\log r} .
$$

Then for almost every $x \in X$, we have

$$
\underline{R}(x) \leq \underline{d}_{\mu}(x) \quad \underset{6}{\text { and }} \quad \bar{R}(x) \leq \bar{d}_{\mu}(x) .
$$


Roughly speaking, this theorem asserts that for typical $x \in X$ and for small $r$, the first return time of $x$ in $B(x, r)$ is at most $r^{-d_{\mu}(x)}$. Since $\underline{d}_{\mu}(x) \leq$ $\mathcal{H}_{a}(X)$ for almost every $x \in X$, we can conclude the first part of Theorem 3.4 from Theorem 3.6. For related results the interested reader should consult the survey [6] and the bibliography therein.

We also remark that the previous results and related concepts have been applied to estimate the dimension of certain strange attractors (see 79 and the references therein) and the entropy of certain Gibbsian systems [36.

We end this section with a result that connects "wandering rates" of sets in infinite measure preserving systems with their "recurrence rates". The next theorem follows easily from a result about lower bounds on ergodic averages for measure preserving systems due to Leibman ([94]); a weaker form for conservative, ergodic systems can be found in Aaronson ([2]).

Theorem 3.7. Let $(X, \mathcal{B}, \mu, T)$ be an infinite measure preserving system, and $A \in \mathcal{B}$ with $\mu(A)<\infty$. Then for all $N \in \mathbb{N}$,

$$
\left(\frac{\mu\left(\bigcup_{n=0}^{N-1} T^{-n} A\right)}{N} \cdot \sum_{n=0}^{N-1} \mu\left(A \cap T^{-n} A\right)\right) \geq \frac{1}{2} \cdot(\mu(A))^{2} .
$$

\section{Subsequence Recurrence}

In this section we discuss what restrictions we can impose on the set of return times in the various versions of the Poincaré recurrence theorem. We start with:

Definition 4.1. Let $R \subset \mathbb{Z}$. Then $R$ is a set of:

(a) Recurrence if for any invertible measure preserving system $(X, \mathcal{B}, \mu, T)$, and $A \in \mathcal{B}$ with $\mu(A)>0$, there is some nonzero $n \in R$ such that $\mu(A \cap$ $\left.T^{-n} A\right)>0$.

(b) Topological recurrence if for every compact metric space $(X, d)$, continuous transformation $T: X \rightarrow X$ and every $\varepsilon>0$, there are $x \in X$ and nonzero $n \in R$ such that $d\left(x, T^{n} x\right)<\varepsilon$.

It is easy to check that the existence of a single $n \in R$ satisfying the previous recurrence conditions actually guarantees the existence of infinitely many $n \in R$ satisfying the same conditions. Moreover, if $R$ is a set of recurrence then one can see from existence of some $T$-invariant measure $\mu$ that $R$ is also a set of topological recurrence. A (complicated) example showing that the converse is not true was given by Kriz ([93]).

Before giving a list of examples of sets of (topological) recurrence, we discuss some necessary conditions: A set of topological recurrence must contain infinitely many multiples of every positive integer, as one can see by considering rotations on $\mathbb{Z}_{d}, d \in \mathbb{N}$. Hence, the sets $\{2 n+1, n \in \mathbb{N}\},\left\{n^{2}+1, n \in \mathbb{N}\right\}$, $\{p+2, p$ prime $\}$ are not good for (topological) recurrence. If $\left(s_{n}\right)_{n \in \mathbb{N}}$ is a lacunary sequence (meaning $\liminf \operatorname{in}_{n \rightarrow \infty}\left(s_{n+1} / s_{n}\right)=\rho>1$ ), then one can construct an irrational number $\alpha$ such that $\left\{s_{n} \alpha\right\} \in[\delta, 1-\delta]$ for all large 
$n \in \mathbb{N}$, where $\delta>0$ depends on $\rho$ (see [89] for example). As a consequence, the sequence $\left(s_{n}\right)_{n \in \mathbb{N}}$ is not good for (topological) recurrence. Lastly, we mention that by considering product systems, one can immediately show that any set of (topological) recurrence $R$ is partition regular, meaning that if $R$ is partitioned into finitely many pieces then at least one of these pieces must still be a set of (topological) recurrence. Using this observation, one concludes for example that any union of finitely many lacunary sequences is not a set of recurrence.

We present now some examples of sets of recurrence:

Theorem 4.2. The following are sets of recurrence:

(i) Any set of the form $\bigcup_{n \in \mathbb{N}}\left\{a_{n}, 2 a_{n}, \ldots, n a_{n}\right\}$ where $a_{n} \in \mathbb{N}$. This follows from a finitary version of Szemerédi's theorem.

(ii) Any IP-set, meaning a set that consists of all finite sums of some infinite set ([62]).

(iii) Any difference set $S-S$, meaning a set that consists of all possible differences of some infinite set $S$.

(iv) The set $\{p(n), n \in \mathbb{N}\}$ where $p$ is any nonconstant integer polynomial with $p(0)=0(60,104])$. In fact we only have to assume that the range of the polynomial contains multiples of an arbitrary positive integer, this follows from Theorem 4.3 below.

(v) The set $\{p(n), n \in S\}$, where $p$ is an integer polynomial with $p(0)=0$ and $S$ is any IP-set ([14]).

(vi) The set of values of an admissible generalized polynomial. This class contains in particular the smallest function algebra $G$ containing all integer polynomials having zero constant term and such that if $g_{1}, \ldots, g_{k} \in$ $G$ and $c_{1}, \ldots, c_{k} \in \mathbb{R}$ then $\llbracket \sum_{i=1}^{k} c_{i} g_{i} \rrbracket \in G$, where $\llbracket x \rrbracket=\left[x+\frac{1}{2}\right]$ denotes the integer nearest to $x$ (15]).

(vii) The set of shifted primes $\{p-1, p$ prime $\}$, and the set $\{p+1, p$ prime $\}$ ([104).

(viii) $R=\{[a(1)],[a(2)], \ldots\}$, where $a(x)=x^{c}$ for any $c>0$. This follows from Theorem 4.3 below and standard exponential sum estimates; see also [29] for a more general result regarding Hardy sequences.

(ix) The set of values of a random non-lacunary sequence. More precisely, pick $n \in \mathbb{N}$ independently with probability $b_{n}$ where $0 \leq b_{n} \leq 1$ and $\lim _{n \rightarrow \infty} n b_{n}=\infty$, then the resulting set is almost surely a set of recurrence.

(x) Arbitrary shifts of integers with an even (or odd) number of prime factors and other similar sets with arithmetic structure (449).

Showing that the first three sets are good for recurrence is a straightforward modification of the argument used to prove Theorem 2.1. The other examples require more work.

A criterion of Kamae and Mendés-France ([87]) provides a powerful tool that may be used in many instances to establish that a set $R$ is a set of recurrence. We mention a variation of their result: 
Theorem 4.3 (Kamae \& Mendés-France [87]). Suppose that $R=\left\{a_{1}<\right.$ $\left.a_{2}<\ldots\right\}$ is a subset of $\mathbb{N}$ such that:

(i) The sequence $\left\{a_{n} \alpha\right\}_{n \in \mathbb{N}}$ is uniformly distributed in $\mathbb{T}$ for every irrational $\alpha$.

(ii) The set $R_{m}=\left\{n \in \mathbb{N}: m \mid a_{n}\right\}$ has positive density for every $m \in \mathbb{N}$.

Then $R$ is a set of recurrence.

We sketch a proof for this result. First, recall Herglotz's theorem: if $\left(a_{n}\right)_{n \in \mathbb{Z}}$ is a positive definite sequence, then there is a unique measure $\sigma$ on the torus $\mathbb{T}$ such that $a_{n}=\int e^{2 \pi i n t} d \sigma(t)$. The case of interest to us is $a_{n}=\int_{\mathbb{T}} f(x) \cdot f\left(T^{n} x\right) d \mu$, where $T$ is measure preserving and $f \in L^{\infty}(\mu)$; $\left(a_{n}\right)$ is positive definite, and we call $\sigma=\sigma_{f}$ the spectral measure of $f$.

Let now $(X, \mathcal{B}, \mu, T)$ be a measure preserving system and $A \in \mathcal{B}$ with $\mu(A)>0$. Putting $f=\mathbf{1}_{A}$, one has

$$
\lim _{N \rightarrow \infty} \frac{1}{N} \sum_{n=1}^{N} \int f(x) \cdot f\left(T^{a_{n}} x\right) d \mu=\int_{\mathbb{T}} \lim _{N \rightarrow \infty}\left(\frac{1}{N} \sum_{n=1}^{N} e^{2 \pi i a_{n} t}\right) d \sigma_{f}(t) .
$$

For $t$ irrational the limit inside the integral is zero (by condition $(i)$ ), so the last integral can be taken over the rational points in $\mathbb{T}$. Since the spectral measure of a function orthogonal to the subspace

$$
\mathcal{H}=\overline{\left\{f \in L^{2}(\mu): \text { there exists } k \in \mathbb{N} \text { with } T^{k} f=f\right\}}
$$

has no rational point masses, we can easily deduce that when computing the first limit in (11), we can replace the function $f$ by its orthogonal projection $g$ onto the subspace $\mathcal{H}(g$ is again nonnegative and $g \neq 0$ ). To complete the argument, we approximate $g$ by a function $g^{\prime}$ such that $T^{m} g^{\prime}=g^{\prime}$ for some appropriately chosen $m$, and use condition (ii) to deduce that the limit of the average (10) is positive.

In order to apply Theorem 4.3. one uses the standard machinery of uniform distribution. Recall Weyl's criterion: a real valued sequence $\left(x_{n}\right)_{n \in \mathbb{N}}$ is uniformly distributed $\bmod 1$ if for every non-zero $k \in \mathbb{Z}$,

$$
\lim _{N \rightarrow \infty} \frac{1}{N} \sum_{n=1}^{N} e^{2 \pi i k x_{n}}=0 .
$$

This criterion becomes especially useful when paired with van der Corput's so-called third principal property: if, for every $h \in \mathbb{N},\left(x_{n+h}-x_{n}\right)_{n \in \mathbb{N}}$ is uniformly distributed $\bmod 1$, then $\left(x_{n}\right)_{n \in \mathbb{N}}$ is uniformly distributed mod 1 . Using the foregoing criteria and some standard (albeit nontrivial) exponential sum estimates, one can verify for example that the sets (iv) and (vii) in Theorem 4.2 are good for recurrence.

In light of the connection elucidated above between uniform distribution mod 1 and recurrence, it is not surprising that van der Corput's method has been adapted by modern ergodic theorists for use in establishing recurrence properties directly. 
Theorem 4.4 (Bergelson [8]). Let $\left(x_{n}\right)_{n \in \mathbb{N}}$ be a bounded sequence in a Hilbert space. If

$$
D-\lim _{m \rightarrow \infty}\left(\lim _{N \rightarrow \infty} \frac{1}{N} \sum_{n=1}^{N}\left\langle x_{n+m}, x_{n}\right\rangle\right)=0,
$$

then

$$
\lim _{N \rightarrow \infty}\left\|\frac{1}{N} \sum_{n=1}^{N} x_{n}\right\|=0 .
$$

Let us illustrate how one uses this "van der Corput trick" by showing that $S=\left\{n^{2}: n \in \mathbb{N}\right\}$ is a set of recurrence. We will actually establish the following stronger fact: If $(X, \mathcal{B}, \mu, T)$ is a measure preserving system and $f \in L^{\infty}(\mu)$ is nonnegative and $f \neq 0$ then

$$
\liminf _{N \rightarrow \infty} \frac{1}{N} \sum_{n=1}^{N} \int f(x) \cdot f\left(T^{n^{2}} x\right) d \mu>0 .
$$

Then our result follows by setting $f=\mathbf{1}_{A}$ for some $A \in \mathcal{B}$ with $\mu(A)>0$.

The main idea is one that occurs frequently in ergodic theory; split the function $f$ into two components, one of which contributes zero to the limit appearing in (3), and the other one being much easier to handle than $f$. To do this consider the $T$-invariant subspace of $L^{2}(X)$ defined by

$$
\mathcal{H}=\overline{\left\{f \in L^{2}(\mu): \text { there exists } k \in \mathbb{N} \text { with } T^{k} f=f\right\}} .
$$

Write $f=g+h$ where $g \in \mathcal{H}$ and $h \perp \mathcal{H}$, and expand the average in (3) into a sum of four averages involving the functions $g$ and $h$. Two of these averages vanish because iterates of $g$ are orthogonal to iterates of $h$. So in order to show that the only contribution comes from the average that involves the function $g$ alone, it suffices to establish that

$$
\lim _{N \rightarrow \infty}\left\|\frac{1}{N} \sum_{n=1}^{N} T^{n^{2}} h\right\|_{L^{2}(\mu)}=0 .
$$

To show this we will apply the Hilbert space van der Corput lemma. For given $h \in \mathbb{N}$, we let $x_{n}=T^{n^{2}} h$ and compute

$$
\begin{aligned}
\lim _{N \rightarrow \infty} \frac{1}{N} \sum_{n=1}^{N}\left\langle x_{n+m}, x_{n}\right\rangle & =\lim _{N \rightarrow \infty} \frac{1}{N} \sum_{n=1}^{N} \int T^{n^{2}+2 n m+m^{2}} h \cdot T^{n^{2}} h d \mu \\
& =\lim _{N \rightarrow \infty} \frac{1}{N} \sum_{n=1}^{N} \int T^{2 n m}\left(T^{m^{2}} h\right) \cdot h d \mu .
\end{aligned}
$$

Applying the ergodic theorem to the transformation $T^{2 m}$ and using the fact that $h \perp \mathcal{H}$, we get that the last limit is 0 . This implies (5).

Thus far we have shown that in order to compute the limit in (3) we can assume that $f=g \in \mathcal{H}$ ( $g$ is also nonnegative and $g \neq 0)$. By the definition of $\mathcal{H}$, given any $\varepsilon>0$, there exists a function $f^{\prime} \in \mathcal{H}$ such that $T^{k} f^{\prime}=f^{\prime}$ 
for some $k \in \mathbb{N}$ and $\left\|f-f^{\prime}\right\|_{L^{2}(\mu)} \leq \varepsilon$. Then the limit in (3) is at least $1 / k$ times the limit

$$
\liminf _{N \rightarrow \infty} \frac{1}{N} \sum_{n=1}^{N} \int f(x) \cdot f\left(T^{(k n)^{2}} x\right) d \mu .
$$

Applying the triangle inequality twice we get that this is greater or equal than

$$
\begin{aligned}
\lim _{N \rightarrow \infty} \frac{1}{N} \sum_{n=1}^{N} \int f^{\prime}(x) \cdot f^{\prime}\left(T^{(k n)^{2}} x\right) d \mu-c \cdot \varepsilon & =\int\left(f^{\prime}(x)\right)^{2} d \mu-2 \varepsilon \\
& \geq\left(\int f^{\prime}(x) d \mu\right)^{2}-c \cdot \varepsilon
\end{aligned}
$$

for some constant $c$ that does not depend on $\varepsilon$ (we used that $T^{k} f^{\prime}=f^{\prime}$ and the Cauchy-Schwartz inequality). Choosing $\varepsilon$ small enough we conclude that the last quantity is positive, completing the proof.

\section{Multiple Recurrence}

Simultaneous multiple returns of positive measure sets to themselves were first considered by H. Furstenberg ([59]), who gave a new proof of Szemerédi's theorem $([108])$ on arithmetic progressions by deriving it from the following theorem:

Theorem 5.1 (Furstenberg [59]). Let $(X, \mathcal{B}, \mu, T)$ be a measure preserving system and $A \in \mathcal{B}$ with $\mu(A)>0$. Then for every $k \in \mathbb{N}$, there is some $n \in \mathbb{N}$ such that

$$
\mu\left(A \cap T^{-n} A \cap \cdots \cap T^{-k n} A\right)>0 .
$$

Furstenberg's proof came by means of a new structure theorem allowing one to decompose an arbitrary measure preserving system into component elements exhibiting one of two extreme types of behavior: compactness, characterized by regular, "almost periodic" trajectories, and weak mixing, characterized by irregular, "quasi-random" trajectories. On $\mathbb{T}$, these types of behavior are exemplified by rotations and by the doubling map, respectively. To see the point, imagine trying to predict the initial digit of the dyadic expansion of $T^{n} x$ given knowledge of the initial digits of $T^{i} x, 1 \leq i<n$. We use the case $k=2$ to illustrate the basic idea.

It suffices to show that if $f \in L^{\infty}(\mu)$ is nonnegative and $f \neq 0$, one has

$$
\liminf _{N \rightarrow \infty} \frac{1}{N} \sum_{n=1}^{N} \int f(x) \cdot f\left(T^{n} x\right) \cdot f\left(T^{2 n} x\right) d \mu>0 .
$$

An ergodic decomposition argument enables us to assume that our system is ergodic. As in the earlier case of the squares, we split $f$ into "almost periodic" and "quasi-random" components. Let $\mathcal{K}$ be the closure in $L^{2}$ of the subspace spanned by the eigenfunctions of $T$, i.e. the functions $f \in L^{2}(\mu)$ that satisfy $f(T x)=e^{2 \pi i \alpha} f(x)$ for some $\alpha \in \mathbb{R}$. We write $f=g+h$, where $g \in \mathcal{K}$ and 
$h \perp \mathcal{K}$. It can be shown that $g, h \in L^{\infty}(\mu)$ and $g$ is again nonnegative with $g \neq 0$. We expand the average in (77) into a sum of eight averages involving the functions $g$ and $h$. In order to show that the only non-zero contribution to the limit comes from the term involving $g$ alone, it suffices to establish that

$$
\lim _{N \rightarrow \infty}\left\|\frac{1}{N} \sum_{n=1}^{N} T^{n} g \cdot T^{2 n} h\right\|_{L^{2}(\mu)}=0,
$$

(and similarly with $h$ and $g$ interchanged, and with $g=h$, which is similar). To establish (8), we use the Hilbert space van der Corput lemma on $x_{n}=$ $T^{n} g \cdot T^{2 n} h$. Some routine computations and a use of the ergodic theorem reduce the task to showing that

$$
\text { D- } \lim _{m \rightarrow \infty}\left(\int h(x) \cdot h\left(T^{2 m} x\right) d \mu\right)=0 \text {. }
$$

But this is well known for $h \perp \mathcal{K}$ (in virtue of the fact that for $h \perp \mathcal{K}$ the spectral measure $\sigma_{h}$ is continuous, for example).

We are left with the average (7) when $f=g \in \mathcal{K}$. In this case $f$ can be approximated arbitrarily well by a linear combination of eigenfunctions, which easily implies that given $\varepsilon>0$ one has $\left\|T^{n} f-f\right\|_{L^{2}(\mu)} \leq \varepsilon$ for a set of $n \in \mathbb{N}$ with bounded gaps. Using this fact and the triangle inequality, one finds that for a set of $n \in \mathbb{N}$ with bounded gaps,

$$
\int f(x) \cdot f\left(T^{n} x\right) \cdot f\left(T^{2 n} x\right) d \mu \geq\left(\int f d \mu\right)^{3}-c \cdot \varepsilon
$$

for a constant $c$ that is independent of $\varepsilon$. Choosing $\varepsilon$ small enough, we get (77).

The new techniques developed for the proof of Theorem 5.1 have led to a number of extensions, many of which have to date only ergodic proofs. To expedite discussion of some of these developments, we introduce a definition:

Definition 5.2. Let $R \subset \mathbb{Z}$ and $k \in \mathbb{N}$. Then $R$ is a set of $k$-recurrence if for every invertible measure preserving system $(X, \mathcal{B}, \mu, T)$ and $A \in \mathcal{B}$ with $\mu(A)>0$, there is some nonzero $n \in R$ such that

$$
\mu\left(A \cap T^{-n} A \cap \cdots \cap T^{-k n} A\right)>0 .
$$

The notions of $k$-recurrence are distinct for different values of $k$. An example of a difference set that is a set of 1-recurrence but not a set of 2recurrence was given in [59]; sets of $k$-recurrence that are not sets of $(k+1)$ recurrence for general $k$ were given in [55] $\left(R_{k}=\left\{n \in \mathbb{N}:\left\{n^{k+1} \sqrt{2}\right\} \in\right.\right.$ $[1 / 4,3 / 4]\}$ is such).

Aside from difference sets, the sets of (1-)recurrence given in Theorem 4.2 may well be sets of $k$-recurrence for every $k \in \mathbb{N}$, though this has not been verified in all cases. Let us summarize the current state of knowledge. The following are sets of $k$-recurrence for every $k$ : Sets of the form $\bigcup_{n \in \mathbb{N}}\left\{a_{n}, 2 a_{n}, \ldots, n a_{n}\right\}$ where $a_{n} \in \mathbb{N}$ (this follows from a uniform version 
of Theorem 5.1 that can be found in [17]). Every IP-set ([62]). The set $\{p(n), n \in \mathbb{N}\}$ where $p$ is any nonconstant integer polynomial with $p(0)=0$ ([19]), and more generally, when the range of the polynomial contains multiples of an arbitrary integer (44]). The set $\{p(n), n \in S\}$ where $p$ is an integer polynomial with $p(0)=0$ and $S$ is any IP-set ([22]). The set of values of an admissible generalized polynomial $([24,97])$. Moreover, it was shown in [52] for $k=2$ and in [114] for general $k \in \mathbb{N}$, that the set of shifted primes $\{p-1, p$ prime $\}$, and the set $\{p+1, p$ prime $\}$ are sets of $k$-recurrence (see also [21, 53, 91, 107] for related work). Several other multiple recurrence results were obtain in the last ten years, including results for Hardy sequences $([25,45,466,58])$, various subsets of integer part polynomial sequences $([88,91,92])$, random sequences $([56,57])$, and sets of arithmetic nature $([18,48,49])$.

More generally, one would like to know for which sequences of integers $a_{1}(n), \ldots, a_{k}(n)$ it is the case that for every invertible measure preserving system $(X, \mathcal{B}, \mu, T)$ and $A \in \mathcal{B}$ with $\mu(A)>0$, there is some nonzero $n \in \mathbb{N}$ such that

$$
\mu\left(A \cap T^{-a_{1}(n)} A \cap \cdots \cap T^{-a_{k}(n)} A\right)>0 .
$$

Unfortunately, a criterion analogous to the one given in Theorem 4.3 for 1 -recurrence is not yet available for $k$-recurrence when $k>1$. Nevertheless, there have been some notable positive results, such as the following:

Theorem 5.3 (Bergelson \& Leibman [19]). Let $(X, \mathcal{B}, \mu, T)$ be an invertible measure preserving system and $p_{1}(n), \ldots, p_{k}(n)$ be integer polynomials with zero constant term. Then for every $A \in \mathcal{B}$ with $\mu(A)>0$, there is some $n \in \mathbb{N}$ such that

$$
\mu\left(A \cap T^{-p_{1}(n)} A \cap \cdots \cap T^{-p_{k}(n)} A\right)>0 .
$$

Furthermore, it has been shown that the $n$ in (10) can be chosen from any IP set ([22]), and the polynomials $p_{1}, \ldots, p_{k}$ can be chosen to belong to the more general class of admissible generalized polynomials (97]) or the class of intersective polynomials $([20])$.

An important boost in the area of multiple recurrence was given by a breakthrough of Host and Kra ([80]). Building on work of Conze and Lesigne ([38, 39]) and Furstenberg and Weiss ([66]) (see also the excellent survey [83], exploring close parallels with [71] and the seminal paper of Gowers ([69])), they isolated the structured component (or factor) of a measure preserving system that one needs to analyze in order to prove several multiple recurrence and convergence results. This allowed them, in particular, to prove existence of $L^{2}$ limits for the so-called "Furstenberg ergodic averages" $\frac{1}{N} \sum_{n=1}^{N} \prod_{i=0}^{k} f\left(T^{i n} x\right)$, which had been a major open problem since the original ergodic proof of Szemerédi's theorem. Subsequently Ziegler in [117] gave a new proof of the aforementioned limit theorem and established minimality of the factor in question. It turns out that this minimal component admits of a purely algebraic characterization; it is a nilsystem, i.e. a rotation on a 
homogeneous space of a nilpotent Lie group. This fact, coupled with some recent results about nilsystems (see [95, 96] for example), makes the analysis of some otherwise intractable multiple recurrence problems much more manageable. These developments have made it possible to obtain new multiple recurrence results and they also allowed us to estimate the size of the multiple intersection in (6) for $k=2,3$ (the case $k=1$ is Theorem 3.1):

Theorem 5.4 (Bergelson, Host \& Kra 16]). Let $(X, \mathcal{B}, \mu, T)$ be an ergodic measure preserving system and $A \in \mathcal{B}$. Then for $k=2,3$ and for every $\varepsilon>0$,

$$
\mu\left(A \cap T^{-n} A \cap \cdots \cap T^{-k n} A\right)>\mu^{k+1}(A)-\varepsilon
$$

for a set of $n \in \mathbb{N}$ with bounded gaps.

Based on work of Ruzsa that appears as an appendix to the paper, it is also shown in [16] that a similar estimate fails for ergodic systems (with any power of $\mu(A)$ on the right hand side) when $k \geq 4$. Moreover, when the system is nonergodic it also fails for $k=2,3$, as can be seen with the help of an example in [7]. Again considering the doubling map $T x=2 x$ and the set $A=[0,1 / 2]$, one sees that the positive results for $k \leq 3$ are sharp. When the polynomials $n, 2 n, \ldots, k n$ are replaced by linearly independent polynomials $p_{1}, p_{2}, \ldots, p_{k}$ with zero constant term, similar lower bounds hold for every $k \in \mathbb{N}$ without assuming ergodicity ([54). The case where the polynomials $n, 2 n, 3 n$ are replaced with general polynomials $p_{1}, p_{2}, p_{3}$ with zero constant term is treated in 44] (see also [40]) and more general results involving Hardy field sequences and polynomials evaluated at the primes are obtained in [40].

\section{Connections with Combinatorics and Number Theory}

The combinatorial ramifications of ergodic-theoretic recurrence were first observed by Furstenberg, who perceived a correspondence between recurrence properties of measure preserving systems and the existence of structures in sets of integers having positive upper density. This gave rise to the field of ergodic Ramsey theory, in which problems in combinatorial number theory are treated using techniques from ergodic theory. The following formulation is from $[9]$.

Theorem 6.1. Let $\Lambda$ be a subset of the integers. There exists an invertible measure preserving system $(X, \mathcal{B}, \mu, T)$ and a set $A \in \mathcal{B}$ with $\mu(A)=\bar{d}(\Lambda)$ such that

$$
\bar{d}\left(\Lambda \cap\left(\Lambda-n_{1}\right) \cap \ldots \cap\left(\Lambda-n_{k}\right)\right) \geq \mu\left(A \cap T^{-n_{1}} A \cap \cdots \cap T^{-n_{k}} A\right),
$$

for all $k \in \mathbb{N}$ and $n_{1}, \ldots, n_{k} \in \mathbb{Z}$.

Proof. The space $X$ will be taken to be the sequence space $\{0,1\}^{\mathbb{Z}}, \mathcal{B}$ is the Borel $\sigma$-algebra, while $T$ is the shift map defined by $(T x)(n)=x(n+1)$ for $x \in\{0,1\}^{\mathbb{Z}}$, and $A$ is the set of sequences $x$ with $x(0)=1$. So the only thing that depends on $\Lambda$ is the measure $\mu$ which we now define. For $m \in \mathbb{N}$ set 
$\Lambda^{0}=\mathbb{Z} \backslash \Lambda$ and $\Lambda^{1}=\Lambda$. Using a diagonal argument we can find an increasing sequence of integers $\left(N_{m}\right)_{m \in \mathbb{N}}$ such that $\lim _{m \rightarrow \infty}\left|\Lambda \cap\left[1, N_{m}\right]\right| / N_{m}=\bar{d}(\Lambda)$ and such that

$$
\lim _{m \rightarrow \infty} \frac{\left|\left(\Lambda^{i_{1}}-n_{1}\right) \cap\left(\Lambda^{i_{2}}-n_{2}\right) \cap \cdots \cap\left(\Lambda^{i_{r}}-n_{r}\right) \cap\left[1, N_{m}\right]\right|}{N_{m}}
$$

exists for every $n_{1}, \ldots, n_{r} \in \mathbb{Z}$, and $i_{1}, \ldots, i_{r} \in\{0,1\}$. For $n_{1}, n_{2}, \ldots, n_{r} \in \mathbb{Z}$, and $i_{1}, i_{2}, \ldots, i_{r} \in\{0,1\}$, we define the measure $\mu$ of the cylinder set $\left\{x_{n_{1}}=\right.$ $\left.i_{1}, x_{n_{2}}=i_{2}, \ldots, x_{n_{r}}=i_{r}\right\}$ to be the limit (13). Thus defined, $\mu$ extends to a premeasure on the algebra of sets generated by cylinder sets and hence by Carathéodory's extension theorem $([35])$ to a probability measure on $\mathcal{B}$. It is easy to check that $\mu(A)=\bar{d}(\Lambda)$, the shift transformation $T$ preserves the measure $\mu$ and (12) holds.

Using this principle for $k=1$, one may check that any set of recurrence is intersective, that is intersects $E-E$ for every set $E$ of positive density. Using it for $n_{1}=n, n_{2}=2 n, \ldots, n_{k}=k n$, together with Theorem 5.1. one gets an ergodic proof of Szemerédi's theorem ([108]), stating that every subset of the integers with positive upper density contains arbitrarily long arithmetic progressions (conversely, one can easily deduce Theorem 5.1 from Szemerédi's theorem, and that intersective sets are sets of recurrence). Making the choice $n_{1}=n^{2}$ and using part $(i v)$ of Theorem 4.3, we get an ergodic proof of the surprising result of Sárközy ([104]) stating that every subset of the integers with positive upper density contains two elements whose difference is a perfect square. More generally, using Theorem 6.1, one can translate all of the recurrence results of the previous two sections to results in combinatorics. (This is not straightforward for Theorem 5.4 because of the ergodicity assumption made there. We refer the reader to [16] for the combinatorial consequence of this result.) We mention explicitly only the combinatorial consequence of Theorem 5.3.

Theorem 6.2 (Bergelson \& Leibman [19]). Let $\Lambda \subset \mathbb{Z}$ with $\bar{d}(\Lambda)>0$, and $p_{1}, \ldots, p_{k}$ be integer polynomials with zero constant term. Then $\Lambda$ contains infinitely many configurations of the form $\left\{x, x+p_{1}(n), \ldots, x+p_{k}(n)\right\}$.

The ergodic proof is the only one known for this result, even for patterns of the form $\left\{x, x+n^{2}, x+2 n^{2}\right\}$ or $\left\{x, x+n, x+n^{2}\right\}$.

Ergodic-theoretic contributions to the field of geometric Ramsey theory were made by Furstenberg, Katznelson, and Weiss (65]), who showed that if $E$ is a positive upper density subset of $\mathbb{R}^{2}$ then: $(i) E$ contains points with any large enough distance (see also [30] and [43]), (ii) Every $\delta$-neighborhood of $E$ contains three points forming a triangle congruent to any given large enough dilation of a given triangle (in [30 it is shown that if the three points lie on a straight line one cannot always find three points with this property in $E$ itself). Recently, a generalization of property ( $i i)$ to arbitrary finite configurations of $\mathbb{R}^{m}$ was obtained by Ziegler ([118]). 
We also mention some exciting connections of multiple recurrence with some structural properties of the set of prime numbers. The first one is in the work of Green and Tao ([71]), where the existence of arbitrarily long arithmetic progressions of primes was demonstrated, the authors, in addition to using Szemerédi's theorem outright, use several ideas from its ergodictheoretic proofs, as appearing in [59] and 64]. The second one is in the recent work of Tao and Ziegler [111, a quantitative version of Theorem 5.3 was used to prove that the primes contain arbitrarily long polynomial progressions. Furthermore, results in ergodic theory related to the structure of the minimal characteristic factors of certain multiple ergodic averages, play an important role in the work of Green, Tao, and Ziegler ([72, 73, 74, 75, 76]), were they get asymptotic formulas for the number of $k$-term arithmetic progressions of primes up to $x$. This work verifies an interesting special case of the Hardy-Littlewood $k$-tuple conjecture predicting the asymptotic growth rate of $N_{a_{1}, \ldots, a_{k}}(x)=$ the number of configurations of primes having the form $\left\{p, p+a_{1}, \ldots, p+a_{k}\right\}$ with $p \leq x$.

In a more recent development, the tools developed in the last two decades to deal with delicate multiple recurrence problems have played an instrumental role in analyzing the structure of measure-preserving systems naturally associated with bounded multiplicative functions. These results were used in the last two years in works of Tao and Teräväinen ([109, 110]) to make progress on the Chowla and Elliott conjectures and in works of Frantzikinakis and Host $([50,51])$ to make progress on the Möbius disjointness conjecture of Sarnak. It appears that this interplay of ergodic theory and number theory is going to be essential for the resolution of several notoriously difficult problems concerning higher order correlations of multiplicative and other number theoretic functions.

Finally, we remark that in this article we have restricted attention to multiple recurrence and Furstenberg correspondence for $\mathbb{Z}$ actions, while in fact there is a wealth of literature on extensions of these results to general commutative, amenable and even non-amenable groups. For an excellent exposition of these and other recent developments the reader is referred to the survey articles [1, 10, 12, 13. Here, we give just one notable combinatorial corollary to some work of this kind, a density version of the classical HalesJewett coloring theorem ([78]).

Theorem 6.3 (Furstenberg \& Katznelson [63]; see also [102]). Let $W_{n}(A)$ denote the set of words of length $n$ with letters in the alphabet $A=\left\{a_{1}, \ldots, a_{k}\right\}$. For every $\varepsilon>0$ there exists $N_{0}=N_{0}(\varepsilon, k)$ such that if $n \geq N_{0}$ then any subset $S$ of $W_{n}(A)$ with $|S| \geq \varepsilon k^{n}$ contains a combinatorial line, i.e., a set consisting of $k$-letter words, having fixed letters in $l$ positions, for some $0 \leq l<n$, the remaining $n-l$ positions being occupied by a variable letter $x$, for $x=a_{1}, \ldots, a_{k}$. (For example, in $W_{4}(A)$ the sets $\left\{\left(a_{1}, x, a_{2}, x\right): x \in A\right\}$ and $\{(x, x, x, x),: x \in A\}$ are combinatorial lines.) 
At first glance, the uninitiated reader may not appreciate the importance of this "master" density result, so it is instructive to derive at least one of its immediate consequences. Let $A=\{0,1, \ldots, k-1\}$ and interpret $W_{n}(A)$ as integers in base $k$ having at most $n$ digits. Then a combinatorial line in $W_{n}(A)$ is an arithmetic progression of length $k$-for example, the line $\left\{\left(a_{1}, x, a_{2}, x\right): x \in A\right\}$ corresponds to the progression $\{m, m+n, m+$ $2 n, m+3 n\}$, where $m=a_{1}+a_{2} d^{2}$ and $n=d+d^{3}$. This allows one to deduce Szemerédi's theorem. Similarly, one can deduce from Theorem 6.3 multidimensional and IP extensions of Szemerédi's theorem ([61, 62]), and some related results about vector spaces over finite fields ([62]).

\section{Future Directions}

In this section we formulate a few open problems relating to the material in the previous three sections. It should be noted that this selection reflects the authors' interests, and does not strive for completeness. A more extensive list of problems related to ergodic theory of $Z$-actions can be found in [47.

We start with an intriguing question of Katznelson ([89]) about sets of topological recurrence. A set $S \subset \mathbb{N}$ is a set of Bohr recurrence if for every $\alpha_{1}, \ldots, \alpha_{k} \in \mathbb{R}$ and $\varepsilon>0$ there exists $s \in S$ such that $\left\{s \alpha_{i}\right\} \in[0, \varepsilon] \cup[1-\varepsilon, 1)$ for $i=1, \ldots, k$.

Problem 1. Is every set of Bohr recurrence a set of topological recurrence?

Background for this problem and evidence for a positive answer can be found in [89, 113]. A negative for a related question concerning general Abelian groups is given in [77]. As we mentioned in Section 4, there exists a set of topological recurrence (and hence Bohr recurrence) that is not a set of recurrence.

Problem 2. Is the set $S=\left\{l ! 2^{m} 3^{n}: l, m, n \in \mathbb{N}\right\}$ a set of recurrence? Is it a set of $k$-recurrence for every $k \in \mathbb{N}$ ?

It can be shown that $S$ is a set of Bohr recurrence. Theorem 4.3 cannot be applied since the uniform distribution condition fails for some irrational numbers $\alpha$. A relevant question was asked by Bergelson in [10]: "Is the set $S=\left\{2^{m} 3^{n}: m, n \in \mathbb{N}\right\}$ good for single recurrence for weakly mixing systems?"

We mentioned in Section 4 that the sets of shifted primes and the set of fractional powers of integers are known to be sets of $k$-recurrence for every $k \in \mathbb{N}$. The following related question remains open:

Problem 3. Show that for every $c \in \mathbb{R}^{+} \backslash \mathbb{Z}$ the set $\left\{\left[p^{c}\right], p \in \mathbb{P}\right\}$, where $\mathbb{P}$ is the set of primes, is a set of $k$-recurrence for every $k \in \mathbb{N}$.

The problem is open even for $k=2$ and $c=\frac{3}{2}$.

We mentioned in Section 4 that random non-lacunary sequences (see definition there) are almost surely sets of recurrence. 
Problem 4. Show that random non-lacunary sequences are almost surely sets of $k$-recurrence for every $k \in \mathbb{N}$.

Some progress is made in [26, 32, 33, 37, 56, 57] but the problem is open even for $k=2$ for random sequences with at most quadratic growth. We refer the reader to the survey [103] for a nice exposition of the argument used by Bourgain [31] to handle the case $k=1$.

It was shown in [55] that if $S$ is a set of 2-recurrence then the set of its squares is a set of recurrence for circle rotations. The same method shows that it is actually a set of Bohr recurrence.

Problem 5. If $S \subset \mathbb{Z}$ is a set of 2-recurrence, is it true that $S^{2}=\left\{s^{2}: s \in\right.$ $S\}$ is a set of recurrence?

A similar question was asked in [34]: "If $\mathrm{S}$ is a set of $k$-recurrence for every $k$, is the same true of $S^{2}$ ?".

One would like to find a criterion that would allow one to deduce that a sequence is good for double (or higher order) recurrence from some uniform distribution properties of this sequence.

Problem 6. Find necessary conditions for double recurrence similar to the one given in Theorem 4.3 .

It is now well understood that such a criterion should involve uniform distribution properties of some generalized polynomials or 2-step nilsequences.

We mentioned in Section 6 that every positive density subset of $\mathbb{R}^{2}$ contains points with any large enough distance. Bourgain ([30]) constructed a positive density subset $E$ of $\mathbb{R}^{2}$, a triangle $T$, and numbers $t_{n} \rightarrow \infty$, such that $E$ does not contain congruent copies of all $t_{n}$-dilations of $T$. But the triangle $T$ used in this construction is degenerate, which leaves the following question open:

Problem 7. Is it true that every positive density subset of $\mathbb{R}^{2}$ contains a triangle congruent to any large enough dilation of a given non-degenerate triangle?

For further discussion on this question the reader can consult the survey [70].

The following question of Aaronson and Nakada (2]) is related to a classical question of Erdôs concerning whether every $K \subset \mathbb{N}$ such that $\sum_{n \in K} 1 / n=\infty$ contains arbitrarily long arithmetic progressions:

Problem 8. Suppose that $(X, \mathcal{B}, \mu, T)$ is a $\{1 / n\}$-conservative ergodic measure preserving system. Is it true that for every $A \in \mathcal{B}$ with $\mu(A)>0$ and $k \in \mathbb{N}$ we have $\mu\left(A \cap T^{-n} A \cap \cdots \cap T^{-k n} A\right)>0$ for some $n \in \mathbb{N}$ ?

The answer is positive for the class of Markov shifts, and it is remarked in [2] that if the Erdôs conjecture is true then the answer will be positive in general. The converse is not known to be true. For a related result showing that multiple recurrence is preserved by extensions of infinite measure preserving systems see [98]. 
Our next problem is motivated by the question whether Theorem 6.3 has a polynomial version (for a precise formulation of the general conjecture see [10]). Not even this most special consequence of it is known to hold.

Problem 9. Let $\varepsilon>0$. Does there exist $N=N(\varepsilon)$ having the property that every family $P$ of subsets of $\{1, \ldots, N\}^{2}$ satisfying $|P| \geq \varepsilon 2^{N^{2}}$ contains a configuration $\{A, A \cup(\gamma \times \gamma)\}$, where $A \subset\{1, \ldots, N\}^{2}$ and $\gamma \subset\{1, \ldots, N\}$ with $A \cap(\gamma \times \gamma)=\emptyset$ ?

A measure preserving action of a general countably infinite group $G$ is a function $g \rightarrow T_{g}$ from $G$ into the space of measure preserving transformations of a probability space $X$ such that $T_{g h}=T_{g} T_{h}$. It is easy to show that a version of Khintchine's recurrence theorem holds for such actions: if $\mu(A)>$ 0 and $\varepsilon>0$ then $\left\{g: \mu\left(A \cap T_{g} A\right)>(\mu(A))^{2}-\varepsilon\right\}$ is syndetic. However it is unknown whether the following ergodic version of Roth's theorem holds.

Problem 10. Let $\left(T_{g}\right)$ and $\left(S_{g}\right)$ be measure preserving $G$-actions of a probability space $X$ that commute in the sense that $T_{g} S_{h}=S_{h} T_{g}$ for all $g, h \in$ $G$. Is it true that for all positive measure sets $A$, the set of $g$ such that $\mu\left(A \cap T_{g} A \cap S_{g} A\right)>0$ is syndetic?

We remark that for general (possibly amenable) groups $G$ not containing arbitrarily large finite subgroups nor elements of infinite order, it is not known whether one can find a single such $g \neq e$. On the other hand, the answer is known to be positive for general $G$ in case $\left(T_{g}^{-1} S_{g}\right)$ is a $G$-action ([23]); even under such strictures, however, it is unknown whether a triple recurrence theorem holds.

\section{REFERENCES}

[1] T. Austin. Multiple Recurrence and Finding Patterns in Dense Sets. In D. Badziahin, A. Gorodnik, \& N. Peyerimhoff (Eds.), Dynamics and Analytic Number Theory (London Mathematical Society Lecture Note Series, pp. 189-257). Cambridge: Cambridge University Press.

[2] J. Aaronson. The asymptotic distribution behavior of transformations preserving infinite measures. J. Analyse Math., 39, (1981), 203-234.

[3] J. Aaronson. An introduction to infinite ergodic theory. Mathematical Surveys and Monographs, 50, American Mathematical Society, Providence, RI, (1997).

[4] J. Aaronson, H. Nakada. Multiple recurrence of Markov shifts and other infinite measure preserving transformations. Israel J. Math., 117, (2000), 285-310.

[5] L. Barreira. Hausdorff dimension of measures via Poincaré recurrence. Comm. Math. Phys., 219, (2001), 443-463.

[6] L. Barreira. Poincaré recurrence: old and new. XIVth International Congress on Mathematical Physics, World Sci. Publ., Hackensack, NJ, (2005), 415-422.

[7] F. Behrend. On sets of integers which contain no three in arithmetic progression. Proc. Nat. Acad. Sci., 23, (1946), 331-332.

[8] V. Bergelson. Weakly mixing PET. Ergodic Theory Dynam. Systems, 7, (1987), 337-349.

[9] V. Bergelson. Ergodic Ramsey Theory. Logic and Combinatorics (editted by S. Simpson). Contemporary Mathematics 65, (1987), 63-87. 
[10] V. Bergelson. Ergodic Ramsey Theory - an update, Ergodic Theory of $\mathbb{Z}^{d}$-actions (edited by M. Pollicott and K. Schmidt). London Math. Soc. Lecture Note Series, 228, (1996), 1-61.

[11] V. Bergelson. The multifarious Poincare recurrence theorem. Descriptive set theory and dynamical systems (ed. M. Foreman, A. Kechris, A. Louveau, B. Weiss), London Math. Soc. Lecture Note Series, 277, (2000), 31-57.

[12] V. Bergelson. Combinatorial and Diophantine Applications of Ergodic Theory. Appendix A by A. Leibman and Appendix B by A. Quas and M. Wierdl, Handbook of Dynamical Systems, vol. 1B, (edited by B. Hasselblatt and A. Katok), Elsevier (2006), 745-841.

[13] V. Bergelson. Ergodic Ramsey Theory: a dynamical approach to static theorems. Proceedings of the International Congress of Mathematicians, Madrid 2006, Vol. II, 1655-1678.

[14] V. Bergelson, H. Furstenberg, R. McCutcheon. IP-sets and polynomial recurrence. Ergodic Theory Dynam. Systems, 16, (1996), 963-974.

[15] V. Bergelson, I. Håland-Knutson, R. McCutcheon. IP Systems, generalized polynomials and recurrence. Ergodic Theory Dynam. Systems, 26, (2006), 999-1019.

[16] V. Bergelson, B. Host, B. Kra, with an appendix by I. Ruzsa. Multiple recurrence and nilsequences. Inventiones Math., 160, 2, (2005), 261-303.

[17] V. Bergelson, B. Host, R. McCutcheon, F. Parreau. Aspects of uniformity in recurrence. Colloq. Math., 84/85, (2000), part 2, 549-576.

[18] V. Bergelson, J. Kułaga-Przymus, M. Lemańczyk. A structure theorem for level sets of multiplicative functions and applications. To appear in Int. Math. Res. Not., arXiv: 1708.02613

[19] V. Bergelson, A. Leibman. Polynomial extensions of van der Waerden's and Szemerédi's theorems. J. Amer. Math. Soc., 9, (1996), 725-753.

[20] V. Bergelson, A. Leibman, E. Lesigne. Intersective polynomials and the polynomial Szemerédi theorem. Adv. Math., 219, (2008), no. 1, 369-388.

[21] V. Bergelson, A. Leibman, T. Ziegler. The shifted primes and the multidimensional Szemerédi and polynomial van der Waerden Theorems. Comptes Rendus Mathematique, 349, (2011), no. 3-4, 123-125.

[22] V. Bergelson, R. McCutcheon. An ergodic IP polynomial Szemerédi theorem. Mem. Amer. Math. Soc., 146, (2000), viii+106 pp.

[23] V. Bergelson, R. McCutcheon. Central sets and a noncommutative Roth theorem. Amer. J. Math., 129, (2007), 1251-1275.

[24] V. Bergelson, R. McCutcheon. Idempotent ultrafilters, multiple weak mixing and Szemerédi's theorem for generalized polynomials. J. Analyse Math., 111, (2010), $77-130$.

[25] V. Bergelson, J. Moreira, F.K. Richter Single and multiple recurrence along nonpolynomial sequences. Preprint, arXiv:1711.05729

[26] B. Bhattacharya, S. Ganguly, X. Shao, Y. Zhao. Upper tails large deviations for arithmetic progressions in a random set. To appear in Int. Math. Res. Not., arXiv: 1605.02994

[27] G. Birkhoff. A proof of the ergodic theorem. Proc. Nat. Acad. Sci., 17, (1931), 656-660.

[28] M. Boshernitzan. Quantitative recurrence results. Invent. Math., 113, (1993), 617631.

[29] M. Boshernitzan, G. Kolesnik, A. Quas, M. Wierdl. Ergodic averaging sequences. J. Analyse Math. 95 (2005), 63-103.

[30] J. Bourgain. A Szemerédi type theorem for sets of positive density in $\mathbb{R}^{k}$. Israel J. Math. 54, no. 3, (1986), 307-316.

[31] J. Bourgain. On the maximal ergodic theorem for certain subsets of the positive integers. Israel J. Math., 61, (1988), 39-72. 
[32] J. Briët, Z. Dvir, S. Gopi. Outlaw distributions and locally decodable codes. Proceedings of ITCS, (2017), arXiv:1609.06355

[33] J. Briët, S. Gopi. Gaussian width bounds with applications to arithmetic progressions in random settings. To appear in Int. Math. Res. Not., arXiv:1711.05624

[34] T. Brown, R. Graham, B. Landman. On the set of common differences in van der Waerden's theorem on arithmetic progressions. Canad. Math. Bull., 42, (1999), $25-36$.

[35] C. Carathéodory. Vorlesungen über reelle Funktionen. 3rd edition, Chelsea Publishing Co., New York, (1968).

[36] J. Chazottes, E. Ugalde. Entropy estimation and fluctuations of hitting and recurrence times for Gibbsian sources. Discrete Contin. Dyn. Syst. Ser. B, 5, no. 3 (2005), 565-586.

[37] M. Christ. On random multilinear operator inequalities. Preprint, arXiv: 1108.5655 .

[38] J. Conze, E. Lesigne. Théorèmes ergodiques pour des mesures diagonales. Bull. Soc. Math. France, 112, no. 2, (1984), 143-175.

[39] J. Conze, E. Lesigne. Sur un théorème ergodique pour des mesures diagonales. Probabilités, Publ. Inst. Rech. Math. Rennes, 1987-1, Univ. Rennes I, Rennes, (1988), $1-31$.

[40] S. Donoso, A.N. Le, J. Moreira, W. Sun. Optimal lower bounds for multiple recurrence. Preprint, arXiv:1809.06912

[41] M. Einsiedler, T. Ward. Ergodic theory with a view towards number theory. Graduate Texts in Mathematics, 259, Springer-Verlag London, Ltd., London, 2011.

[42] D. Evans, D. Searles. The fluctuation theorem. Advances in Physics, 51, (2002), 1529-1585.

[43] K. Falconer, J. Marstrand. Plane sets with positive density at infinity contain all large distances. Bull. Lond. Math. Soc., 18, (1986), 471-474.

[44] N. Frantzikinakis. Multiple ergodic averages for three polynomials and applications. Trans. Amer. Math. Soc., 360, (2008), no. 10, 5435-5475.

[45] N. Frantzikinakis. Equidistribution of sparse sequences on nilmanifolds. J. Analyse Math., 109, (2009), 353-395.

[46] N. Frantzikinakis. Multiple recurrence and convergence for Hardy sequences of polynomial growth. J. Analyse Math. 112 (2010), 79-135.

[47] N. Frantzikinakis. Some open problems on multiple ergodic averages. Bull. Hellenic Math. Soc., 60 (2016), 41-90.

[48] N. Frantzikinakis, B. Host. Higher order Fourier analysis of multiplicative functions and applications. J. Amer. Math. Soc., 30, (2017), 67-157.

[49] N. Frantzikinakis, B. Host. Multiple ergodic theorems for arithmetic sets. Trans. Amer. Math. Soc., 369, no. 10, (2017), 7085-7105.

[50] N. Frantzikinakis, B. Host. The logarithmic Sarnak conjecture for ergodic weights. Ann. of Math. 187 (2018), 869-931.

[51] N. Frantzikinakis, B. Host. Furstenberg systems of bounded multiplicative functions and applications. To appear in Int. Math. Res. Not. IMRN, arXiv:1804.08556

[52] N. Frantzikinakis, B. Host, B. Kra. Multiple recurrence and convergence for sets related to the primes. J. Reine Angew. Math., 611, (2007), 131-144.

[53] N. Frantzikinakis, B. Host, B. Kra. The polynomial multidimensional Szemerédi Theorem along shifted primes. Isr. J. Math., 194, (2013), 331-348.

[54] N. Frantzikinakis, B. Kra. Ergodic averages for independent polynomials and applications. J. London Math. Soc., 74, Issue 1, (2006), 131-142.

[55] N. Frantzikinakis, E. Lesigne, M. Wierdl. Sets of $k$-recurrence but not $(k+1)$ recurrence. Annales de l'Institut Fourier, 56, Issue 4, (2006), 839-849.

[56] N. Frantzikinakis, E. Lesigne, M. Wierdl. Random sequences and pointwise convergence of multiple ergodic averages. Indiana Univ. Math. J., 61, (2012), 585-617. 
[57] N. Frantzikinakis, E. Lesigne, M. Wierdl. Random differences in Szemerédi's theorem and related results. J. Analyse Math., 130, (2016), 91-133.

[58] N. Frantzikinakis, M. Wierdl. A Hardy field extension of Szemerédi's theorem. Adv. Math., 222, (2009), 1-43.

[59] H. Furstenberg. Ergodic behavior of diagonal measures and a theorem of Szemerédi on arithmetic progressions. J. d'Analyse Math, 71, (1977), 204-256.

[60] H. Furstenberg. Recurrence in ergodic theory and combinatorial number theory. Princeton University Press, Princeton, (1981).

[61] H. Furstenberg, Y. Katznelson. An ergodic Szemerédi theorem for commuting transformations. J. Analyse Math., 34, (1979), 275-291.

[62] H. Furstenberg, Y. Katznelson. An ergodic Szemerédi theorem for IP-systems and combinatorial theory. J. Analyse Math., 45, (1985), 117-168.

[63] H. Furstenberg, Y. Katznelson. A density version of the Hales-Jewett theorem. J. Analyse Math., 57, (1991), 64-119.

[64] H. Furstenberg, Y. Katznelson, D. Ornstein. The ergodic theoretical proof of Szemerédi's theorem. Bull. Amer. Math. Soc. (N.S.), 7, (1982), no. 3, 527-552.

[65] H. Furstenberg, Y. Katznelson, B. Weiss. Ergodic theory and configurations in sets of positive density. Mathematics of Ramsey theory, Algorithms Combin., 5, Springer, Berlin, (1990), 184-198.

[66] H. Furstenberg, B. Weiss. A mean ergodic theorem for $(1 / N) \sum_{n=1}^{N} f\left(T^{n} x\right)$ $g\left(T^{n^{2}} x\right)$. Convergence in ergodic theory and probability (Columbus, OH, 1993), Ohio State Univ. Math. Res. Inst. Publ., 5, de Gruyter, Berlin, (1996), 193-227.

[67] S. Galatolo, D. H. Kim, K. K. Park. The recurrence time for ergodic systems with infinite invariant measures. Nonlinearity, 19, (2006), 2567-2580

[68] E. Glasner. Ergodic theory via joinings. Mathematical Surveys and Monographs 101. American Mathematical Society, Providence, RI, 2003.

[69] W. Gowers, A new proof of Szemerédi's theorem. Geom. Funct. Anal., 11, (2001), $465-588$

[70] R. L. Graham. Recent trends in Euclidean Ramsey theory. Trends in discrete mathematics, Discrete Math., 136, (1994), no. 1-3, 119-127.

[71] B. Green, T. Tao. The primes contain arbitrarily long arithmetic progressions. Ann. of Math. 167, (2008), 481-547.

[72] B. Green, T. Tao. Linear equations in primes. Ann. of Math., 171, (2010), 17531850 .

[73] B. Green, T. Tao. The quantitative behaviour of polynomial orbits on nilmanifolds. Ann. of Math., 175, (2012), 465-540.

[74] B. Green, T. Tao. On the quantitative distribution of polynomial nilsequenceserratum. Ann. of Math., 179, (2014), 1175-1183, arXiv:1311.6170v3

[75] B. Green, T. Tao. The Möbius function is strongly orthogonal to nilsequences. Ann. of Math., 175, (2012), 541-566.

[76] B. Green, T. Tao, T. Ziegler. An inverse theorem for the Gowers $U^{s+1}[N]$-norm. Ann. of Math., 176, (2012), no. 2, 1231-1372.

[77] J. Griesmer. Bohr topology and difference sets for some abelian groups. Preprint, arXiv: 1608.01014

[78] A. Hales, R. Jewett. Regularity and positional games. Trans. Amer. Math. Soc. 106, (1963), 222-229.

[79] T. Hasley, M. Jensen. Hurricanes and butterflies. Nature, 428, (2004), 127-128.

[80] B. Host, B. Kra. Nonconventional ergodic averages and nilmanifolds. Ann. of Math., 161, (2005), 397-488.

[81] B. Host, B. Kra. Nilpotent Structures in Ergodic Theory. Mathematical Surveys and Monographs, vol. 236. American Mathematical Society, Providence, RI, 2018. 
[82] M. Kac. On the notion of recurrence in discrete stochastic processes. Bull. Amer. Math. Soc., 53, (1947), 1002-10010.

[83] B. Kra. The Green-Tao theorem on arithmetic progressions in the primes: an ergodic point of view. Bull. Amer. Math. Soc. (N.S.), 43, (2006), 3-23.

[84] B. Kra. From combinatorics to ergodic theory and back again. Proceedings of International Congress of Mathematicians, Madrid 2006, Vol. III, 57-76.

[85] B. Kra. Ergodic methods in additive combinatorics. Additive combinatorics, 103143, CRM Proc. Lecture Notes, 43, Amer. Math. Soc., Providence, RI, 2007.

[86] B. Kra. Poincare recurrence and number theory: thirty years later. Bull. Amer. Math. Soc., 48, (2011), 497-501.

[87] T. Kamae, M. Mendés-France. Van der Corput's difference theorem. Isr. J. Math., 31, (1978), 335-342.

[88] D. Karageorgos, A. Koutsogiannis. Integer part independent polynomial averages and applications along primes. To appear in Studia Mathematica, arXiv: 1708.06820

[89] Y. Katznelson. Chromatic numbers of Cayley graphs on $\mathbb{Z}$ and recurrence. Paul Erdös and his mathematics (Budapest, 1999), Combinatorica, 21, (2001), no. 2, $211-219$

[90] A. Khintchine. Eine Verschärfung des Poincaréschen "Wiederkehrsatzes". Comp. Math., 1, (1934), 177-179.

[91] A. Koutsogiannis. Closest integer polynomial multiple recurrence along shifted primes. Ergodic Theory Dynam. Systems, 38, (2018), 666-685.

[92] A. Koutsogiannis. Integer part polynomial correlation sequences. Ergodic Theory Dynam. Systems, 38, (2018), 1525-1542.

[93] I. Kriz, Large independent sets in shift invariant graphs. Solution of Bergelson's problem. Graphs and Combinatorics, 3, (1987), 145-158.

[94] A. Leibman. Lower bounds for ergodic averages. Ergodic Theory Dynam. Systems, 22, (2002), 863-872.

[95] A. Leibman. Pointwise convergence of ergodic averages for polynomial sequences of rotations of a nilmanifold. Ergodic Theory Dynam. Systems, 25, (2005), 201-213.

[96] A. Leibman. Pointwise convergence of ergodic averages for polynomial actions of $\mathbb{Z}^{d}$ by translations on a nilmanifold. Ergodic Theory Dynam. Systems, 25, (2005), $215-225$.

[97] R. McCutcheon. FVIP systems and multiple recurrence. Israel J. Math., 146, (2005), 157-188.

[98] T. Meyerovitch. On multiple and polynomial recurrent extensions of infinite measure preserving transformations. Unpublished. Available at arXiv:0703914v2.

[99] D. Ornstein and B. Weiss. Entropy and data compression schemes. IEEE Trans. Inform. Theory, 39, (1993), 78-83.

[100] K. Petersen. Ergodic theory. Cambridge Studies in Advanced Mathematics, 2, Cambridge University Press, Cambridge, (1989).

[101] H. Poincaré. Sur le problème des trois corps et les équations de la dynamique. Acta Math., 13, (1890), 1-270.

[102] D. H. J. Polymath. A new proof of the density Hales-Jewett theorem. Ann. of Math., 175, (2012), 1283-1327.

[103] J. Rosenblatt, M. Wierdl. Pointwise ergodic theorems via harmonic analysis. Ergodic theory and its connections with harmonic analysis (Alexandria, 1993). London Math. Soc. Lecture Note Ser., 205, Cambridge Univ. Press, Cambridge, (1995), 3-151.

[104] A. Sárközy. On difference sets of integers III. Acta Math. Acad. Sci. Hungar., 31, (1978), 125-149.

[105] I. Shkredov. Recurrence in the mean. Mat. Zametki, 72, (2002), no. 4, 625-632; translation in Math. Notes, 72, (2002), no. 3-4, 576-582. 
[106] L. Sklar. Philosophy of Statistical Mechanics. The Stanford Encyclopedia of Philosophy (Summer 2004 Edition), Edward N. Zalta (ed.), URL = $\langle$ http://plato.stanford.edu/archives/sum2004/ entries/statphys-statmech/〉.

[107] W. Sun. Multiple recurrence and convergence for certain averages along shifted primes. Ergodic Theory Dynam. Systems 35 (2015), no. 5, 1592-1609.

[108] E. Szemerédi. On sets of integers containing no $k$ elements in arithmetic progression. Acta Arith., 27, (1975), 299-345.

[109] T. Tao, J. Teräväinen. The structure of logarithmically averaged correlations of multiplicative functions, with applications to the Chowla and Elliott conjectures. To appear in Duke Math. J., arXiv:1708.02610

[110] T. Tao, J. Teräväinen. The structure of correlations of multiplicative functions at almost all scales, with applications to the Chowla and Elliott conjectures. Preprint, arXiv: 1809.02518

[111] T. Tao, T. Ziegler. The primes contain arbitrarily long polynomial progressions. Acta Math., 201, (2008), 213-305.

[112] P. Walters. An introduction to ergodic theory. Graduate Texts in Mathematics, 79, Springer-Verlag, New York-Berlin, (1982).

[113] B. Weiss. Single orbit dynamics. CBMS Regional Conference Series in Mathematics, 95, American Mathematical Society, Providence, RI, (2000).

[114] T. Wooley, T. Ziegler. Multiple recurrence and convergence along the primes. Amer. J. of Math., 134, (2012), 1705-1732.

[115] A. Wyner, J. Ziv. Some asymptotic properties of the entropy of a stationary ergodic data source with applications to data compression. IEEE Trans. Inform. Theory, 35, (1989), 1250-1258.

[116] E. Zermelo. Über einen Satz der Dynamik und die mechanische Wärmetheorie, Annalen der Physik, 57, (1896), 485-94; English translation On a Theorem of Dynamics and the Mechanical Theory of Heat, in Kinetic Theory, ed. S. G. Brush (Oxford, 1966), II, 208-17.

[117] T. Ziegler. Universal characteristic factors and Furstenberg averages. J. Amer. Math. Soc., 20, (2007), 53-97.

[118] T. Ziegler. Nilfactors of $\mathbb{R}^{m}$-actions and configurations in sets of positive upper density in $\mathbb{R}^{m}$. J. Anal. Math., 99, (2006), 249-266.

(Nikos Frantzikinakis) University of Crete, Department of Mathematics, Voutes University Campus, Heraklion 71003, Greece

E-mail address: frantzikinakis@gmail.com

(Randall McCutcheon) University of Memphis, Department of Mathematics, Memphis, TN 38152-3240, USA

E-mail address: rmcctchn@memphis.edu 